\title{
On Statistics of the Mobile Rayleigh Fading Channel in Non-Isotropic Scattering Environments
}

\author{
Rauf Iqbal and Thushara D. Abhayapala \\ Department of information Engineering \\ Research School of Information Sciences and Engineering \\ The Australian National University, ACT 200, AUSTRALIA \\ Tel: +61-2-6125-6246, Fax: +61-2-6230-6121 \\ E-mails: rauf.iqbal@anu.edu.au;thushara.abhayapala@anu.edu.au
}

\begin{abstract}
Scattering encountered in many wireless communications scenarios is non-isotropic. Assumption of uniform distribution of Power Azimuth Spectrum (PAS) in a non-isotropic scattering environment introduces significant errors on the second order channel statistics which are the basis for the estimation of some important receiver parameters. Moreover, there are certain applications in communications that rely solely on the statistics of the channel. In this contribution, we use the wellknown Jacobi-Anger expansion of the plane wave to develop a discrete-time generalized Rayleigh fading channel model that models the statistics of the channel in closed form in general, nonisotropic and isotropic, scattering environments. We compare the statistics of the channel for different commonly used non-isotropic scattering distributions, first, on the basis of autocorrelation, and, then, using a function (mutual information) of the correlational properties of the channel. In the latter case, through simulations, we observe the effect of varying different parameters like the angular spread, the block length of transmission and the mobile velocity which gives some interesting insights.
\end{abstract}

\section{INTRODUCTION}

In real world communication scenarios, the transmitter and/or the receiver may be in motion. A mobile-radio situation in which transmitter is fixed in position while the receiver is moving, usually in such a way that the direct line between transmitter and receiver is obstructed by buildings, is more common. At ultra high frequencies and above, therefore, the mode of propagation of the electromagnetic energy from transmitter to receiver is largely by way of scattering [1]. The amplitude fluctuations of the received signal have been shown to follow Rayleigh distribution in this communication scenario. By assuming a uniform power azimuth spectrum (PAS) which corresponds to uniform distribution of the angleof-arrival (AOA) around the receive antenna, the autocorrelation of the received signal was shown to be strictly real valued and the resulting power spectral density (PSD) was U-shaped symmetric.

It has been argued and experimentally demonstrated (see [2] and references therein) that the scattering encountered in many suburban and rural environments is non-isotropic i.e., the distribution of AOA of waves is not uniform as assumed in [1]. Moreover, the use of a directional antenna (in an isotropic scattering environment) with non-uniform gain pattern at the receiver also results in non-isotropic scattering as seen by the antenna. The assumption of PAS introduces small errors on the first order statistics of the received signal but a significant error on the second order statistics, like correlation function or, equivalently, PSD, and level crossing rates or, equivalently, the fading rate.

An adaptive communication receiver can improve the performance and reduce the complexity by using the second order statistics to control receiver parameters, such as pilot filter bandwidth, automatic gain control loop bandwidth, phase tracker bandwidth and interleaver size [3]. The second order statistics are also useful for handoff decisions [4] in cellular communication systems. Moreover, the transmission of information in non-coherent Gaussian fading depends solely on the correlational properties of the received signal. It is, therefore, of some interest to develop a model that accurately models the statistics of the mobile channel in general, isotropic and non-isotropic, scattering environments which is the subject of this contribution.

The effect of angular distribution on the spatial correlation in a multiple-input multiple-output (MIMO) was explored in [5]. The effect of angular power distribution on temporal correlation, however, has not been considered yet. While we consider a single-input single output (SISO) system, the results can be extended to a MIMO system. Using the generalized Rayleigh fading model, we present comparative results for most commonly employed PAS environments based on the same assumptions and geometric references which gives new insights, which were not available before.

The rest of the paper is organized as follows. In section II we develop a generalized discrete time channel model. In section III, we use the information rate penalty function [6] for unknown CSI which depends directly on the statistics of the channel to compare some of the more common non-isotropic scattering distributions. Numerical results are discussed in IV. Finally, the conclusion is drawn in section V.

Throughout the paper, the following notation will be used: Bold lower (upper) letters denote vectors (matrices). ${ }^{*}$ and $T$ denote the conjugate transpose, and transpose, respectively. The symbol $\mathbb{C}$ denotes the unit circle. The log function is the natural logarithm so that the information rates and differential entropies are expressed in nats/symbol. The ceiling operator is denoted by $\lceil$.$\rceil . The notation E\{\cdot\}$ denotes the mathematical expectation. 


\section{CHANNEL MODEL}

We consider a downlink SISO transmission system where the transmitter is stationary while the receiver equipped with isotropic antenna is moving with speed $|\boldsymbol{v}|$ at an angle $\phi_{v}$ with respect to $\mathrm{x}$-axis (Fig.1). We assume that the scatterers are distributed in the far-field from the receive antenna. We also assume that the channel between the transmitter and the mobile receiver is a strictly bandlimited, flat-fading (frequency non-selective), wide-sense stationary, zero mean circularly symmetric Gaussian fading process. We assume, in addition, that the channel output is processed through a unit-energy matched filter at least at the Nyquist rate corresponding to the time-varying channel fading process.

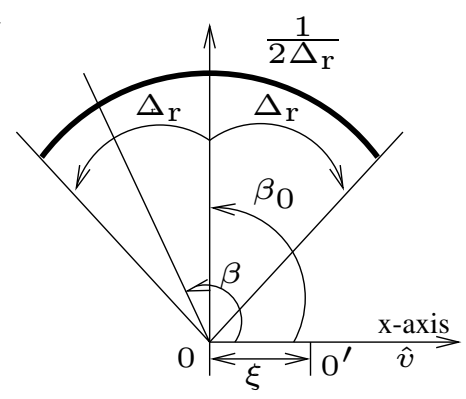

Fig. 1. The Uniform-limited scattering scenario where the scattered power is uniformly distributed over a circular sector with mean scattering angle $\beta_{0}$ and maximum deviation of $\Delta_{r}$ on each side of the mean. For simplicity, the mobile has been shown to be moving with velocity $\boldsymbol{v}$ along positive $\mathrm{x}$-axis with $\phi_{v}=0^{\circ}$.

Following [1] let the mobile moving with velocity $\boldsymbol{v}$ be at some arbitrary point 0 at the signaling instant 0 . Thus, at the signaling interval $j$, the mobile will be at the point $\left(|v| j T_{s}, \phi_{v}\right)$ with respect to 0 , where $\phi_{v}$ is the direction of the mobile with respect to $\mathrm{x}$-axis and $T_{s}$ is the symbol duration. Let $x_{j}$ be the transmitted symbol during the jth signaling interval. Then the complex baseband received signal at the signaling interval $j$ is

$$
y_{j}=\sqrt{\rho} h_{j} x_{j}+n_{j},
$$

where $\left\{n_{j}\right\}$ are samples of an i.i.d zero mean proper complex additive gaussian noise process with unit variance, $\rho$ is the average received signal-to-noise ratio (SNR) per symbol and $h_{j}$ is the channel gain at $j$ th symbol interval given by

$$
h_{j}=\int_{\mathbb{C}} f(\beta) e^{i \eta j \boldsymbol{v} \cdot \hat{\boldsymbol{\beta}}} \mathrm{d} \beta,
$$

where $\eta=2 \pi / \lambda$ is the free space phase constant dependent on the carrier wave length, $\lambda, f(\beta)$ is the effective complex random scattering gain of the received signal from an angle $\beta$ and $\hat{\boldsymbol{\beta}}$ represents the unit vector in the direction of $\beta$.

Using (1) we can write the correlation between the channel gain at the signaling intervals $j^{\prime}$ and $j=j^{\prime}+k$ ( $k$ represents the lag in number of symbols) as

$$
\begin{aligned}
\Phi\left(j, j^{\prime}\right) & =E\left\{h_{j} h_{j^{\prime}}^{*}\right\}, \\
& =\iint_{\mathbb{C}} E\left\{f\left(\beta^{\prime}\right) f^{*}(\beta)\right\} e^{i \eta T_{s}|\boldsymbol{v}|\left(j-j^{\prime}\right) \hat{\boldsymbol{v}} \cdot \hat{\boldsymbol{\beta}}} \mathrm{d} \beta \mathrm{d} \beta^{\prime},
\end{aligned}
$$

where $\hat{\boldsymbol{v}}$ is the unit vector in the direction of mobile direction of travel. Assume that the scattering gains from two distinct directions are uncorrelated and are zero mean [7], i.e.,

$$
E\left\{f(\beta) f^{*}\left(\beta^{\prime}\right\}=E\left\{|f(\beta)|^{2}\right\} \delta\left(\beta-\beta^{\prime}\right) .\right.
$$

Using (4) and making use of the assumption of wide-sense stationarity of the fading process in (3), we can write

$$
\Phi\left(j, j^{\prime}\right)=\Phi\left(\left|j-j^{\prime}\right|\right)=\Phi(k)=\iint_{\mathbb{C}} F(\beta) e^{i \eta T_{s} k|\boldsymbol{v}| \hat{\boldsymbol{v}} \cdot \hat{\boldsymbol{\beta}}} \mathrm{d} \beta,
$$

where $F(\beta)=E\left\{|f(\beta)|^{2}\right\}$ is normally termed as the angular power distribution $[8]$ of the received signal.

Notice that $F(\beta)$ is periodic in $\beta$. It can, therefore, be expanded using a Fourier series with orthogonal circular harmonics as the basis set. We define

$$
\begin{aligned}
\gamma_{n} & =\int_{\mathbb{C}} F(\beta) e^{-i n \beta} \mathrm{d} \beta, \\
F(\beta) & =\frac{1}{2 \pi} \sum_{n=-\infty}^{\infty} \gamma_{n} e^{i n \beta},
\end{aligned}
$$

where $\gamma_{n}$ are the coefficients of the Fourier series expansion of $F(\beta)$. The plane wave $e^{-i \eta k T_{s}|\boldsymbol{v}| \hat{\boldsymbol{v}} \cdot \hat{\boldsymbol{\beta}}}$ can be expanded using Jacobi-Anger expansion [9, page 67] as

$$
e^{i \eta k T_{s}|\boldsymbol{v}| \hat{\boldsymbol{v}} \cdot \hat{\boldsymbol{\beta}}}=\sum_{n=-\infty}^{\infty} i^{n} J_{n}\left(\eta k T_{s}|\boldsymbol{v}|\right) e^{i n\left(\beta-\phi_{v}\right)},
$$

where $\boldsymbol{v}=|\boldsymbol{v}| e^{i \phi_{v}}$ and, $\beta$ and $\phi_{v}$ both are measured with respect to $\mathrm{x}$-axis (Fig. 1).

Let $\omega_{d}=\eta v$ be the maximum angular Doppler spread (maximum Doppler frequency, $\left.f_{d}=|v| / \lambda\right)$. Combining equations (5), (6b) (7), we get

$$
\Phi(k)=\sum_{n=-\infty}^{\infty} i^{n} \gamma_{n} J_{n}\left(\omega_{d} k T_{s}\right) e^{-i n \phi_{v}} .
$$

Equation (8) is the complex autocorrelation function of the channel fading process as a function of lag $k$. As can be observed the autocorrelation of the fading process, in general, not only depends on PAS through $\gamma_{n}$ but also on the direction of mobile travel.

Making use of the well-known Wiener-Khintchine theorem [10], by taking discrete-time Fourier transform (DTFT) of (8), it can be shown that PSD of the channel fading process is given by

$$
\Phi(\omega)=\frac{1}{\omega_{D}} \sum_{n=-\infty}^{\infty} \gamma_{n} e^{-i n \phi_{v}} F_{n}\left(\frac{\omega}{\omega_{D}}\right)
$$


where $\omega \in[-\pi, \pi]$ is the continuous radian frequency variable. Each term in (9) gives the contribution of power of each harmonic of PAS as a function of doppler frequency and may be called Power Modal Spectral Density (PMSD).

Equations (8) and (9) (together with zero mean proper assumption for the fading process) fully characterize the statistics of the channel fading process under general 2D nonisotropic conditions for any PAS environment. It can easily be shown that equations (8) and (9) reduce to the Clarke's case when the angular spread ${ }^{1}$ is equal to that of uniform PAS (isotropic scattering).

The equations (8) and (9), though, involve summation over infinity, it is almost always possible in cases of practical interest to safely truncate the respective series up to a finite number of terms in the light of the following two facts:

i) $\quad$ For a fixed order $n, J_{n}(x)$ starts small and reaches to its maximum at arguments $x \approx O(n)$ before starts decaying slowly. It was shown in [11] that $J_{n}(x) \approx 0$ for $|n|>2\lceil x / 2\rceil+1$ with $e=2.7183 \ldots$ Since the argument of $J_{n}\left(k \omega_{\mathrm{D}}\right)$ depends on the lag variable $k$, the approximation would also depend on $k$ for a fixed normalized fading rate.

ii) The Fourier coefficients $\gamma_{n}$ must decay with $\mathrm{n}$ for Fourier series to be convergent (see e.g., [12]). The rate of decay depends on the smoothness of the function which is, in turn, related to the number of continuous derivatives of the function. In fact, the Fourier coefficients of an analytic (infinitely differentiable) function decay exponentially with $n$. Fourier coefficients of a Gaussian distribution, for example, decay exponentially with $n$, and decay polynomially for Laplace distribution. The Fourier coefficients for a uniform distribution decay as $1 / n$. In all these cases, the Fourier coefficients tend to zero $^{2}$ as $n \rightarrow \infty$. The rapid the decay of the Fourier series coefficients, the less the number of Fourier modes $\left(\gamma_{n}\right)$ with significant contribution and vice versa.

For small $k$, the fact (i) is useful in approximating (8) due to the presence of $J_{n}\left(k \omega_{\mathrm{D}}\right)$. As $\mathrm{k}$ increases, the number of Fourier coefficients that are "allowed" to contribute also increases. In the limit $k \rightarrow \infty$, the number of Fourier modes that contribute to (8) also approaches infinity. This is where the fact (ii) comes into effect. Due to the decay (e.g., exponential for a Gaussian distribution) of $\gamma_{n}$ with $\mathrm{n}$, the values of $\gamma_{n}$ become increasingly small so that there must exist some finite $n_{0}$ such that $\gamma_{n} \approx 0$ for all $|n|>n_{0}$. We can, therefore, truncate the infinite summation in (8) to a summation over $|n|=n_{0}$ terms with almost no approximation error.

\footnotetext{
${ }^{1}$ We define angular spread as the standard deviation of PAS (the pdf of the scattered power distribution). The angular spread of the uniform PAS is $\approx 104^{\circ}$.

${ }^{2}$ The Delta distribution is an exception to this behavior. The Fourier coefficients of a Delta distribution do not tend to zero at all, indicative of the fact that it is not an ordinary function and its Fourier series does not converge in the standard sense.
}

The approximation of the PSD of the fading process in (9) seems difficult due to the presence of the factor $F_{n}\left(\frac{\omega}{\omega_{D}}\right)$ which, for all $n$, sharply increases as $\omega$ approaches $\omega_{D}$ becoming infinite at $\omega_{\mathrm{D}}$. The approximation, therefore, would depend on the rate of decay of the Fourier coefficients of a particular scattering distribution. As long as the decay of $\gamma_{n}$ is sufficiently fast, we can use the fact (ii) to approximate (9) to some finite $|n|=n_{0}^{\prime}$. The accuracy of this approximation, moreover, would depend on the angular spread (standard deviation) of the scattering distribution and the mobile direction of travel.

\section{AppliCATions of the Generalized RAYLEIGH FADING MODEL}

In the work on receiver correlation modelling, [13] has derived Fourier coefficients $\gamma_{n}$ in closed-form for some common non-isotropic scattering distributions. The use of these coefficients $\gamma_{n}$ in generalized Rayleigh fading model ((8) and (9)) enables us to compare and quantify the difference in behavior of common non-isotropic scattering distributions in any scenario where second order statistics are important. One obvious application where comparison of non-isotropic scattering environments might be useful is the scenario of information communication over Rayleigh fading channels when no CSI is available but only second order statistics are available.

In [6], the information rates that can be achieved reliably over Rayleigh fading channel in an isotropic scattering environment without CSI have been shown to depend directly on second order statistics of the channel. It was shown in [6] that the cost, $P_{\delta}$, that has always to be paid in terms of information rate loss when no CSI is available is given by [6]

$$
\begin{aligned}
P_{\delta} & \leq \log \operatorname{det}(\boldsymbol{I}+\rho \boldsymbol{C}), \\
& =\sum_{\ell=1}^{N} \log \left(1+\rho \lambda_{\ell}\right),
\end{aligned}
$$

where quality holds for constant power signaling (M-ary Phase Shift Keying), $N$ is the block length of transmission and $\lambda_{\ell}, \ell=1,2, \cdots, N$, are the eigenvalues of the covariance matrix $C$ of the channel process with $\left(j, j^{\prime}\right)$ entry expressed as

$$
[\boldsymbol{C}]_{j, j^{\prime}}=E\left\{h_{j} h_{j^{\prime}}^{*}\right\}=\Phi(k),
$$

where $k=\left|j-j^{\prime}\right|$ represents the lag and $\Phi(k)$ is given by (8).

We can observe that $P_{\delta}$ depends on the spectrum of the fading process through the eigenvalues, $\lambda_{i}$, of the channel covariance matrix. $P_{\delta}$ represents the mutual information between the received output vector, $\mathbf{y}$ and the channel vector, $\mathbf{h}$, conditioned on the knowledge of the input sequence, $\mathrm{x}$. For the same angular spread, a scattering distribution that imparts harsher second order statistics to the resulting channel process will have higher $P_{\delta}$ and vice versa. In fact $P_{\delta}$ quantifies the difference in second order statistics of different scattering environments in the jargon of classical communication theory. Thus, $P_{\delta}$ is a useful measure that gives a physical meaning to 


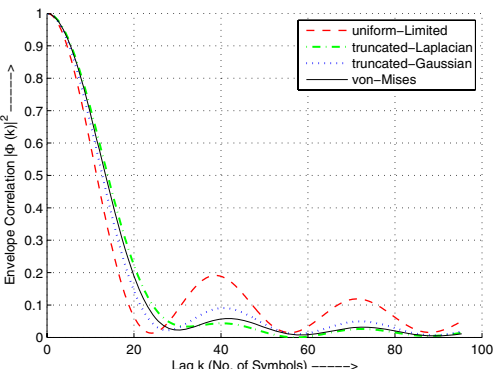

Fig. 2. Autocorrelation of the fading process for different non-isotropic scattering distributions versus the lag $\mathrm{k}$ for $\Lambda=55^{\circ}, f_{\mathrm{D}}=0.05, \phi_{v}=75^{\circ}$ and $\beta_{0}=90^{\circ}$. Only $|n|=25$ terms were sufficient.

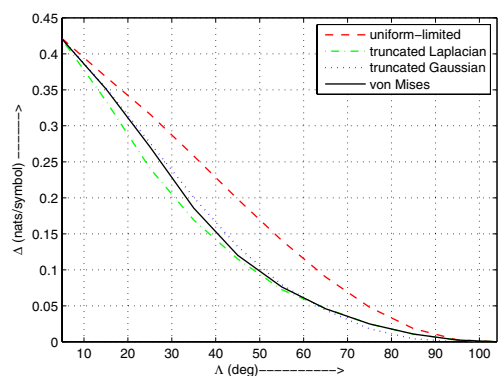

Fig. 3. The behavior of $\Delta$ for common non-isotropic scattering distributions as a function of the angular spread, $\Lambda$ when $f_{\mathrm{D}}=0.05, N=40, \phi_{v}=75^{\circ}$ and $\beta_{0}=90^{\circ}$.

the statistics of the channel and can be used for performance evaluation of a non-coherent communication system, designed to operate in one environment, in different non-isotropic distributions.

Another important application of the generalized Rayleigh fading model is the performance evaluation of various velocity (and, hence, Doppler) estimators in different non-isotropic scattering environments. The performance of different velocity estimators in a non-isotropic Rician fading environment where AOA distribution is modelled by Von-Mises distribution has been evaluated in [4]. The generalized Rayleigh fading model developed here would be useful to quantify the difference in performance of various velocity estimators (designed to operate in a particular environment) in general scattering environments.

\section{NumericAl ANALYSIS AND Discussion}

In this Section, we shall first present some numerical results that would help quantify the effect on the autocorrelation of the channel process of having different non-isotropic scattering distributions. Then, we shall compare $P_{\delta}$ defined in Section III for different scattering scenarios, block lengths and directions of mobile travel with respect to the mean scattering angle.

The autocorrelation of the fading process for different distributions has been plotted in Fig. 2 for $\Lambda=55^{\circ}$ when

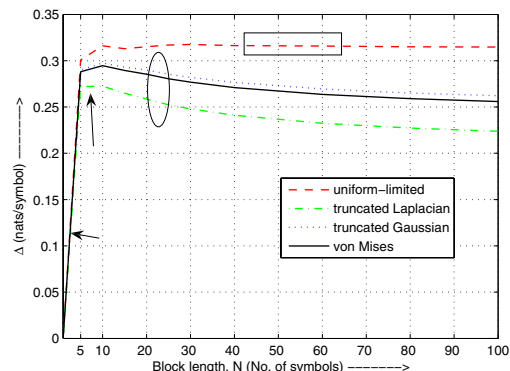

Fig. 4. The behavior of $\Delta$ for common non-isotropic scattering distributions as a function of the block length of transmission $(N)$ when $\Lambda=35^{\circ}, f_{\mathrm{D}}=$ $0.05, \phi_{v}=75^{\circ}$ and $\beta_{0}=90^{\circ}$.

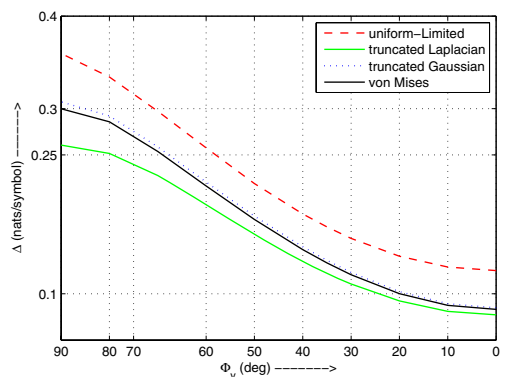

Fig. 5. The behavior of $\Delta$ for common non-isotropic scattering distributions as a function of $\phi_{v}$ when $f_{\mathrm{D}}=0.05, N=40, \Lambda=35^{\circ}$ and $\beta_{0}=90^{\circ}$.

the mobile direction of travel with respect to $\mathrm{x}$-axis is $75^{\circ}$. It is easy to see that uniform-limited scattering distribution has lower correlation than other scattering environments for shorter lags. As the time evolves, the truncated-Gaussian, truncated Laplacian and von-Mises distributions have identical correlations and are "pessimistic" (i.e., have lower correlation) as compared to the Uniform-limited distribution.

For fixed block length and the mobile velocity, Fig. 3 compares $\Delta^{3}=\left(P_{\delta}\right)_{\text {iso }}-\left(P_{\delta}\right)_{\text {non-iso }}$ (where $\left(P_{\delta}\right)_{\text {iso }}$ is the penalty in isotropic environment and $\left(P_{\delta}\right)_{\text {non-iso }}$ is the penalty in non-isotropic scattering environment) as a function of the angular spread. No significant change in this behavior has been observed if the block length is changed. Notice that all common non-isotropic scattering distributions behave similarly (monotonic decrease of $\Delta$ with increasing angular spread) against isotropic environment as a function of the angular spread keeping all other parameters fixed. The autocorrelation behavior shown in Fig. 2 verifies the observation in Fig. 3 that for angular spread of $55^{\circ}$ truncated-Gaussian, truncated Laplacian and von-Mises distributions are identical and less "benign" than uniform-Limited scattering environment. Also notice that for small and very large angular spreads, all non-

${ }^{3}$ According to the definition of $\Delta$, a non-isotropic scattering environment with the lowest $\Delta$ is the "worst" of all non-isotropic environments and vice versa 
isotropic scattering environments are almost identical. For medium to large angular spreads, truncated Laplacian and vonMises distributions remain almost identical (and, therefore, replacement of one distribution for the other is immaterial) but are quite different from uniform-Limited scattering environment.

For a fixed angular spread and the mobile velocity, the difference $\Delta$ has been plotted as a function of block length of transmission in Fig. 4. No significant change in this behavior has been observed if the angular spread is changed. Unlike other non-isotropic scattering distributions, Uniform-limited scattering environment seems to be least sensitive to the change in block length. Also notice that truncated-Laplacian distribution is the "worst" of all the scattering environments as it is closest to the isotropic environment for all block lengths as shown in Fig. 4.

As we have discussed in Section II, the channel statistics in non-isotropic environment, unlike isotropic scattering environment, depend on the mobile direction of travel. The Fig. 5 shows the behavior of $\Delta$ for common non-isotropic scattering distributions as a function $\phi_{v}$ keeping block length of transmission and the angular spread fixed. It is easily observed that the truncated-Laplacian distribution is least sensitive to a change in $\phi_{v}$ as compared to other non-isotropic scattering environments.

\section{CONCLUSION}

A discrete-time generalized channel model using the wellknown Jacobi-Anger expansion was derived that models the Rayleigh fading statistics in general 2D non-isotropic scattering environments (with the 2D isotropic environment as a special case) in closed form. This model can be used to compare and quantify the effect of different non-isotropic scattering environments on the performance of a system designed to operate in a particular scattering environment. Using the generalized Rayleigh fading model we drew comparison between some commonly used non-isotropic scattering environments first on the basis of the autocorrelation and, then, on the basis of a function (mutual information) of the second order statistics of the channel fading process. The comparison of the performances of various velocity (Doppler) estimators in general scattering environments is another important application of the generalized model.

\section{REFERENCES}

[1] R. H. Clarke, "A statistical theory of mobile-radio reception," in Bell System Technical Journal, 1968, vol. 47, pp. 957-1000.

[2] A. Abdi, J.A. Barger, and M. Kaveh, "A parametric model for the distribution of the angle of arrival and the associated correlation function and power spectrum at the mobile station," IEEE Trans. Vehic. Technol., vol. 51, no. 3, pp. 425-434, May 2002.

[3] A. Chockalingam, P. Dietrich, L. B. Milstein, and R. R. Rao, "Performance of closed-loop power control in DS-CDMA cellular systems," IEEE Transactions on Vehicular Technology, vol. 47, no. 3, pp. 774-789, Aug 1998.

[4] C. Tepedelenlioglu and G.B. Giannakis, "On velocity estimation and correlation properties of narrow-band communication channels," IEEE Trans. Vehic. Technol., vol. 50, no. 4, pp. 1039-1052, July 2001.
[5] L. Chan and S. Loyka, "Impact of multipath angular distribution on performance of MIMO systems," Canadian Conference on Electrical and Computer Engineering, CCECE, vol. 2, pp. 853-857, May 2004.

[6] Xinmin Deng and A.M. Haimovich, "Information rates of time varying rayleigh fading channels," in Proc. IEEE International Conference on Communication, ICC'2004, Paris, France, 2004.

[7] J. S. Sadowsky and V. Kafedziski, "On the correlation and scattering functions of the WSSUS channel for mobile communications," in IEEE Transactions on Vehicular Technology, Feb 1998, vol. 47, pp. 270-282.

[8] T. A. Lamahewa, T. D. Abhayapala, R. A. Kennedy, T. Betlehem, and J. T. Y. Ho, "Space-time channel modelling in general scattering environments," IEEE Trans. Sig. Proc., 2006 (submitted).

[9] D. Colton and R. Kress, Inverse Acoustic and Electromagnetic Scattering Theory, vol. 93, Springer, New York, 2 edition, 1998.

[10] D. C. Champeney, A Handbook of Fourier Transforms, Cambridge University Press, U.K., 1987.

[11] H.M. Jones, R.A. Kennedy, and T.D. Abhayapala, "On dimensionality of multipath fields: Spatial extent and richness," in Proc. IEEE Int. Conf Acoust., Speech, Signal Processing, ICASSP'2002, Orlando, Florida, May 2002, vol. 3, pp. 2837-2840.

[12] G. B. Folland, Real Analysis, Wiley-Interscience, New York, ii edition, 1972.

[13] P.D. Teal, T.D. Abhayapala, and R.A. Kennedy, "Spatial correlation for general distributions of scatterers," IEEE Signal Processing Letters, vol. 9, no. 10 , pp. 305-308, Oct 2002. 